\title{
Determination of the Timing Jitter of THz-Synchrotron Radiation by a Cross-Correlation Technique
}

\author{
A. Pohl ${ }^{1,2}$, N. Deßmann ${ }^{1,2}$, A. Hoehl ${ }^{3}$, M. Ries ${ }^{4}$, G. Wüstefeld ${ }^{4}$, G. Ulm ${ }^{3}$, and H.-W. Hübers ${ }^{1,2}$ \\ ${ }^{1}$ Institute of Optical Sensor Systems, German Aerospace Center (DLR), 12489 Berlin, Germany \\ ${ }^{2}$ Humboldt-Universität zu Berlin, Department of Physics, 12489 Berlin, Germany \\ ${ }^{3}$ Physikalisch-Technische Bundesanstalt (PTB), Abbestraße 2-12, 10587 Berlin, Germany \\ ${ }^{4} \mathrm{HZB}$ für Materialien und Energie, Albert-Einstein-Str. 15, 12489 Berlin, Germany
}

\begin{abstract}
The pulse jitter of $\mathrm{THz}$ synchrotron radiation has been derived from the analysis of broadband cross-correlation measurements performed at the synchrotron Metrology Light Source. The pulse jitter is extracted by comparing equivalent autocorrelation measurements recorded with identical beam optics. A pulse jitter of 1.1 ps has been found.
\end{abstract}

\section{INTRODUCTION}

$\mathrm{T}$ HE objective of this study is to determine the timing jitter of $\mathrm{THz}$ synchrotron radiation [1]. The jitter at the beamline is a composition of several instabilities related to the electron beam and the beam optics. By comparing pulses through correlation techniques it is possible to determine the jitter without the need for direct pulse timing measurements [2].

\section{SETUP \& RESUlTS}

Cross-correlation and autocorrelation measurements have been performed at the $\mathrm{THz}$ beamline of the Metrology Light Source (MLS). Providing broadband $\mathrm{THz}$ pulses by the emission of coherent synchrotron radiation (CSR) with a typical repetition rate of $500 \mathrm{MHz}$ and pulse durations in the 1 to $15 \mathrm{ps}$ range the MLS offers great opportunities for beam diagnostics. Aiming for the investigation of the timing jitter of the $\mathrm{THz}$ pulses a dedicated experimental setup comprising a Martin-Puplett interferometer (MPI) and a Fourier transform infrared spectrometer (FTIR) has been established [3, 4]. The MPI generates a correlation signal depending on the optical delay between the $\mathrm{THz}$ pulses. The optical path difference of the MPI was set by a delay line with a step size of $133 \mathrm{fs}$. After passing the MPI the correlation signals were spectroscopically analyzed by a FTIR. Fig. 1 shows the frequency resolved cross-correlation between $\mathrm{THz}$ pulses emitted from two subsequent electron bunches. The vertical axis shows the single frequency correlations versus the optical delay. An appropriate emission spectrum of the beamline ranging from $0.1 \mathrm{THz}$ to $1.1 \mathrm{THz}$ is shown on the left. Dips in the signal at $0.55 \mathrm{THz}$ and $0.75 \mathrm{THz}$ are induced by water vapor absorption. The bunch current was about $1.8 \mathrm{~mA}$. Despite the broad coherent emission spectrum emitted by a single electron bunch, frequencies above $0.3 \mathrm{THz}$ were suppressed in the cross-correlation signal. This is due to the pulse jitter that acts as a low-pass filter when superimposing two successive $\mathrm{THz}$ pulses. The jitter dependent crosscorrelation signal at zero-delay is given by the intensities $I_{1,2}(\omega, t)$ of the vertical and horizontal polarization components

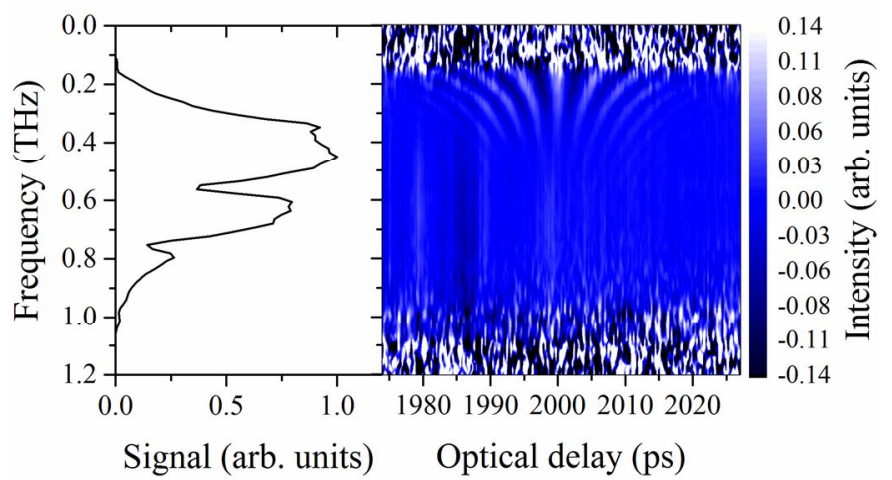

Fig. 1. Left: spectral coverage of the coherent $\mathrm{THz}$ emission during the measurement. Right: Frequency resolved cross-correlation of two successive $\mathrm{THz}$ pulses in the coherent radiation regime. Due to the pulse jitter the correlation is suppressed above $\mathrm{f}_{\mathrm{G}} \approx 275 \mathrm{GHz}$.

$$
\mathbf{I}_{1,2}(\omega, t)=\frac{I_{0}(\omega)}{2}\left(1 \pm \exp \left(-\frac{\left(t-\Delta t_{1,2}\right)^{2}}{2 \sigma^{2}}\right) \cos \left(\omega\left(t-\Delta t_{1,2}\right)\right)+C,\right.
$$

where $\Delta t$ is the jitter of the THz pulses and $\sigma$ the pulse width assuming a Gaussian electron distribution in the longitudinal direction of the electron bunch.

Fig. 2 displays a simulation of the cross-correlation signal at zero delay for different values of the jitter. The spectral envelope of the cross-correlation signal clearly shows the lowpass behavior depending on the jitter of the single bunches.

A single pulse jitter of approximately $1.1 \mathrm{ps}$ was found by curve fitting of the measured cross-correlation signal to the model. The jitter was found to be constant within a bunch current range from $1.8 \mathrm{~mA}$ down to $7.5 \mu \mathrm{A}$.

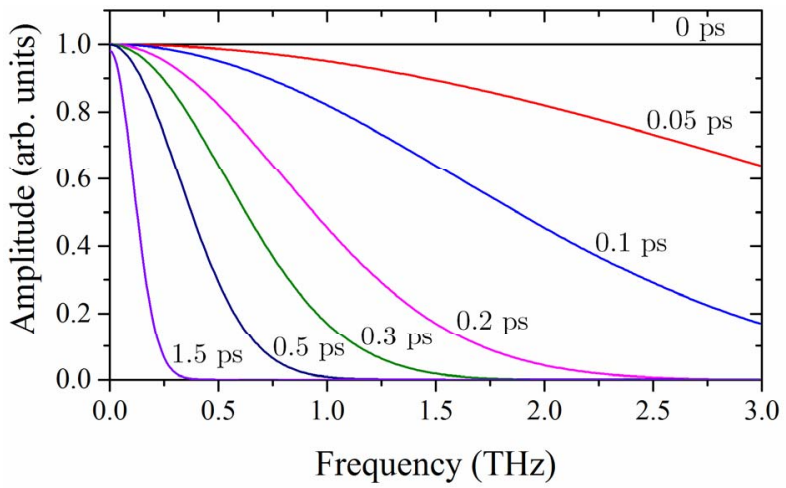

Fig. 2. Influence of random phase jitter on a simulated cross-correlation signal. An increasing jitter leads to a decreasing cut-off frequency. 


\section{ACKNOWLEDGEMENTS}

N. Deßmann and A. Pohl acknowledge the support by the Helmholtz Research School on Security Technologies.

\section{REFERENCES}

[1]. J. Feikes et al., "Metrology Light Source: The first electron storage ring optimized for generating coherent $\mathrm{THz}$ radiation", Phys. Rev. ST Accel. Beams. 14, 030705, 2011.

[2]. A. Pohl, "Untersuchung der THz-Strahlcharakteristik an der Synchrotronstrahlquelle MLS", Dissertation, 2016.

[3]. A. Pohl et al., "Field transients of coherent terahertz synchrotron radiation accessed via time-resolving and correlation techniques," J. Appl. Phys., vol. 119, no. 11, p. 114903, Mar. 2016.

[4]. A. Pohl et al., "THz autocorrelation measurements at the Metrology Light Source," in 2014 39th International Conference on Infrared, Millimeter, and Terahertz waves (IRMMW-THz), 2014. 